\title{
Poésie et utopie en France, au XIXe siècle
}

\author{
Roi de passion une erreur d'optique n'est pas pour \\ altérer la netteté ou réduire l'envergure de ton regard \\ Le calendrier à ton mur a pris toutes les couleurs du \\ spectre.
}

André Breton, Ode à Charles Fourier ${ }^{1}$

L'utopie est avant tout connue en tant que discours, et on la classe alors parmi les « utopismes » (Trousson, 1998). On perçoit aussi en elle une dominante narrative, en référence à l'ouvrage archétypal de Thomas More (Racault, 1991). Les transpositions dramatiques de la pensée sociale, qu'elles soient sincères ou parodiques, sont moins représentées et moins connues que leurs deux principales versions qui viennent d’être évoquées ${ }^{2}$. Rares sont les travaux portant sur le lyrisme de l'avenir et l'enthousiasme réformiste ${ }^{3}$. Par ailleurs, les recherches spécialisées sur la transition entre les utopies

- Françoise Sylvos - professeur de littérature française à l'Université de la Réunion. Adresse de correspondance : Faculté des Lettres et des sciences humaines, Casier 74, Université de La Réunion, 15 avenue René Cassin, 97715 Saint-Denis Messag. Cedex 09, Ile de La Réunion ; e-mail: francoise.sylvos@ univ-reunion.fr

ORCID iD : https://orcid.org/0000-0001-8750-9165

1. Breton, 1999, p. 104.

2. On citera par exemple pour les reprises comiques de l'utopisme au théâtre le vaudeville La propriété c'est le vol de Clairville et Cordier, Théâtre du vaudeville le 28/11/1848, commenté dans Sylvos (2014), "Introduction » du numéro en ligne de la revue Tropics consacrée au thème "Théâtre et utopie », p. 9. La pièce a été analysée en profondeur dans Sylvos (2015). On trouve au contraire une adaptation sérieuse de l'anarchisme dans La Clairière (1900) de Lucien Descaves et Maurice Donnay, étudiée dans Sylvos (2014), p. 10.

3. Voir De Liefde (1927) et Nathan (1981). 
du XVIII siècle et Jules Verne ont eu encore peu d'écho. Dans la perspective d'une recherche novatrice, qui s'inscrit au cœur d'un panorama des genres de l'utopie et de l'anticipation au XIX ${ }^{e}$ siècle, il importe donc de faire connaître ce chaînon manquant de la première moitié du XIX ${ }^{e}$ siècle et d'en montrer les prolongements en fin de siècle .

L'utopie est présente tout au long du XIX ${ }^{e}$ siècle en tant que discours, drame, poésie et narration. Utopies, anticipations ou tableaux futuristes, dystopies, voyages extraordinaires, uchronies et proses lyriques traversent le XIX ${ }^{\mathrm{e}}$ siècle. On s'intéressera ici aux affinités entre l'utopie et la poésie. Il conviendrait dans un premier temps de dépasser les stéréotypes qui réduisent la poésie utopique à sa fonction didactique. Un corpus digne d'intérêt pour l'usage d'un style et d'une langue raffinés, flamboyants, se dessine avec la rétro-SF pathétique et vibrante de Cousin de Grainville (Le dernier homme, 1805), avec les extrapolations cosmiques et paraboles mystiques de Charles Fourier, la prose lyrique de Charles Duveyrier (La ville nouvelle ou le Paris des saint-simoniens, 1832) ou encore la prose biblique de Félicité de Lamennais (Paroles d'un croyant, 1834), avec le caractère visionnaire des légendes et discours hugoliens $\left(X X^{e} s i e ̀ c l e^{5}\right)$, les poèmes aux accents parnassiens inclus dans l'épopée intergalactique de Defontenay (Star ou Psi de Cassiopée, 1854) ${ }^{6}$.

La poésie didactique, à laquelle appartient l'œuvre de Maxime Du Camp (18221894), auteur des Chants modernes (1855), représente le courant des textes de propagande sociale qui comprend encore des corpus peu fréquentés - notamment ceux de la poésie ouvrière.

Plus qu'une écriture soignée, musicale, témoignant de la puissance du Verbe et du pouvoir évocateur de l'image, les textes prophétiques des réformateurs sociaux sont poétiques en tant qu'ils envisagent l'univers comme le lieu d'une double révélation, cachée dans les hiéroglyphes cosmiques et dans les rapports ou accords vibratoires qui en scellent la perfection ${ }^{7}$. La poéticité des utopies du XIX ${ }^{e}$ siècle tient aussi à la re-création du monde (Enfantin, 1832). " Changer la vie » (Rimbaud), rendre la cité " harmonieuse " (Péguy), telle est la vocation des réformateurs qui se proposent de mettre l'esthétique, l'art des effets au service de leur cause. La perfection de leur monde se mire dans leur art, visionnaire. Aussi les mondes qu'ils inventent sont-ils en eux-mêmes des poèmes et sont-ils magnifiés, sublimés par des comparaisons avec la beauté féminine, un idéal, un tableau, une symphonie. L’analogie entre

4. L'essayisme n'est pas la seule forme que prend la pensée sociale au XIX ${ }^{\mathrm{e}}$ siècle. Nombreux sont alors les romans, relations de voyage ou descriptions utopiques, anticipations comme en témoignent quelques exemples : utopies balzaciennes du Médecin de campagne et du Curé de village, voyage en ballon dans un refuge pyrénéen (Mosneron de Launay, Le vallon aérien, Chaumerot, 1810) ou sauts dans un lointain futur (Bodin, Le roman de l'avenir, Lecointe et Pougin, 1834). Pour un panorama complet des formes et enjeux des utopies à cette période, se reporter à Sylvos (2008) et Sylvos (2021).

5. Il s'agit d'une section de La légende des siècles.

6. Le caractère poétique de cette épopée intergalactique a été abordé dans Sylvos (2006).

7. La dimension symbolique des utopies sociales a été abordée dans Sylvos (2007). 
la perfection de la cité et l'harmonie formelle sera au centre de cette étude. La société idéale met en jeu des qualités esthétiques inédites dans les contrées chimériques rêvées par les artistes romantiques. L'impossible devient la règle, dans ces mondes autres.

Le sujet abordé ici outrepasse, on le voit, les limites communément assignées à un article. Nos choix sont motivés par le désir de renouveler la critique en abordant des pans d'un domaine encore méconnu. Après la poésie sociale, l'article abordera la prose inspirée des saint-simoniens Barthélémy Prosper Enfantin (1796-1864) et Charles Duveyrier (1803-1866).

\section{Poèmes didactiques}

"L'industrie, faisant irruption dans l'art, en devient la plus mortelle ennemie... La poésie et le progrès sont deux ambitieux qui se haïssent d'une haine instinctive ", écrit Baudelaire dans « Le public moderne et la photographie » (1868, p. 261). D'emblée, le critique et poète souligne l'incompatibilité entre le modernisme et l'art, entre le didactisme de la propagande positive et la poésie. Baudelaire fustige, à la suite d'Edgar Poe (1887, p. 311), «l'hérésie de l'enseignement ${ }^{8}$; il perçoit un écart radical entre ces discours versifiés et la poésie. Or, paradoxalement, on a montré que le XIX ${ }^{e}$ siècle proposait une somme abondante de poèmes philosophiques et didactiques comprenant des épopées humanitaires généralement placées sous le signe du progrès (Combe, 2005). De l'Orphée de Ballanche (1827-1829) aux Destinées de Vigny (1864, posthume), cette production aujourd'hui tombée en désuétude n'est désormais abordée que par des spécialistes de la littérature du XIX siècle.

Le poète moderne doit privilégier le goût et la recherche du rythme par rapport à la vérité. Or le poème didactique allégorise le progrès dans un style ampoulé qui escamote le réel et le sens. L'image et la construction métaphorique dominent dans des textes qui amplifient et ressassent des thèmes convenus tels que la fécondation de l'avenir ou les lumières du progrès. Ces discours grandiloquents deviennent pain bénit pour ironistes et caricaturistes (Caraion, 2016). De plus, compte tenu des exigences de la modernité, la longueur du poème didactique contribue à sa dépréciation. Enfin, le stéréotype didactique ne saurait correspondre à l'esprit de spontanéité qui caractérise la poésie selon les romantiques.

Les Chants modernes de Maxime Du Camp ont des traits communs avec la réclame. L'éloge de la technique - et par exemple de la vapeur et de la locomotive y est répétitif et ne souffre aucune modulation. Les connotations du discours sur la machine et les nouvelles énergies ne sont en aucun cas nuancées. Le simplisme du jugement sur l'essor des inventions industrielles et mécaniques n'a d'égal que celui de la mise en scène. Le lyrisme des voix poétiques humaines est remplacé par

8. Charles Baudelaire, Notes nouvelles sur Edgar Poe, cité par Combe (2005), p. 63. 
la subjectivité des machines prenant la parole pour se décrire à la première personne. Le goût des comparaisons entre l'humain et les nouvelles machines manque de sûreté. L'apologie du matérialisme technique s'apparente à un discours de propagande.

Chanter les merveilles de la science et de l'industrie devient, au cours du XIX ${ }^{\mathrm{e}}$ siècle, un thème plus topique qu'utopique. À la grande différence des écrivains romantiques, qui clament leur indépendance, nuancent fortement les doctrines sociales et dialoguent avec elles, le poème de propagande progressiste asservit la poésie à la pensée positive - qui avait été formulée dès 1803 par Saint-Simon dans les Lettres d'un habitant de Genève à ses contemporains. En 1863, dans Paris au XXe siècle, Jules Verne regrette l'inféodation des Lettres aux Sciences. Ces vers caricaturaux insérés dans le roman d'anticipation ne sont pas sans évoquer les Chants modernes de Du Camp :

Le charbon porte alors sa flamme incendiaire

Dans les tubes ardents de lénorme chaudière!

Le monstre surchauffé ne craint pas de rivaux !

La machine rugit sous sa tremblante écorce,

Et, tendant sa vapeur, développe une force

De quatre-vingts chevaux.

Mais de son lourd levier le chauffeur vient contraindre

Les tiroirs à s'ouvrir, et dans l'épais cylindre,

Rapide et gémissant, court le double piston!

La roue a patiné ! La vitesse s'active ! Le sifflet part !...

Salut à la locomotive du système Crampton. (Verne, 1863, p. 54)

Le poème s'apparente à une commande d'État motivée par l'académisme en des temps où scientifiques et techniciens deviennent les références en termes de goût. La sensibilité et la liberté individuelle de création ne sont plus de mise. Les poètes indépendants sont condamnés à l'insuccès et à la misère, à l'instar de Michel, personnage principal de Paris au XX $X^{e}$ siècle.

Les poètes positifs et ouvriers s'adonnent parfois à la création collective. Le partage de l'espace livresque renvoie au communautarisme qui anime les réformateurs sociaux dans les Cent et une petites misères, ceuvre sociale (Gille, Letac et Berthier, 1846) et le Banquet des travailleurs socialistes (1849). Le premier ouvrage revisite la formule des " guirlandes " précieuses, œuvres collectives, mais sur des thèmes et dans un style populaires. Toutes les pièces ont en commun la chute «ça doit bien l'gêner su'l moment ». Les apocopes du refrain témoignent d'une langue populaire

9. "Le câble ", repose sur un parallèle pesant. D’abord serpent, le câble est le pont métaphorique entre la suspension des ouvriers qui descendent dans la mine (I) et, dans la deuxième partie du texte, la dépendance affective du sujet (Du Camp, 1860, p. 264). Le poème a beau être versifié, la comparaison accentue le prosaïsme de l'amour. 
qui s'assume. On rencontre parmi ces textes variés des vers anticléricaux, des scènes de l'industrie et du commerce, comme en témoigne par exemple un poème consacré à un accident de chemin de fer :

Sur un ch'min de fer un'machin'neuve
Un jour éclat'dans un'épreuve
L'représentant d'l'autorité
Dans un'mare à cent pas est j'té.
R'peché l'lend'main l'pauvr'commissaire
Pour dresser s'lon son ministère
L'procès-verbal de l'événement
Çà d'vait bien l'gêner su'l' moment.

Louis Guémied (Gille, Letac et Berthier, 1846, p. 8)

Nombreuses sont les catégories socio-professionnelles, ouvrières et bourgeoises, à être représentées dans cet ouvrage panoramique qui réunit agents de change, contremaitres, souffleurs, etc. Ainsi, le recueil propose une fresque réaliste de la vie du peuple se focalisant sur les incidents et accidents du quotidien, sur l'oppression sauvage exercée par les maîtres, sur les services rendus à la France par les soldats conscrits et invalides - , au péril de leur vie et de leur intégrité physique, que ce soit en Europe ou en Orient. Les textes se distinguent par la précision du vocabulaire du monde du travail : "pignon ", pièce mécanique, évoquée par Eugène Berthier (1846, p. 9), "seringue » et " clystère » cités dans un texte du même auteur (1846, p. 27), "guillotine " et " paratonnerre " mentionnés dans un octet de Charles Gille (1846, p. 29). Les textes proposent très fréquemment une variante burlesque des mythes païens et anecdotes bibliques, qui dénote l'instruction des participants. La gaudriole et les thèmes de la complainte populaire (crimes passionnels, accidents mortels, vengeances cruelles, exécutions capitales) y sont omniprésents. Le caractère « social» de ce corpus versifié tient avant tout à la revendication d'une identité culturelle jusqu'alors minorée. Son originalité repose sur la fantaisie, sur le piquant de situations tragi-comiques.

Certains poètes et représentants du peuple s'approprient une forme didactique propice à la vulgarisation des connaissances techniques, la fable ${ }^{10}$. Anarchistes et radicaux exaltent la pensée et la poésie révolutionnaires. Quant aux nouveaux prophètes, ils voient dans ce didactisme empesé une inspiration " sublime $»^{11}$, " fille de l'Evangile et de la philosophie ». Pierre Lachambeaudie (1806-1872) est un fabuliste et chansonnier saint-simonien dont le parcours semble correspondre aux attentes de Saint-Simon dans son appel aux artistes, aux savants et aux industriels de 1824 .

10. Discours de Blanqui cité dans Banquet des travailleurs socialistes, monographie imprimée, 1849.

11. "Toast » du citoyen Salières, cité dans Banquet des travailleurs socialistes, monographie imprimée, 1849, p. 4. 
Le fondateur du saint-simonisme préconisait l'abandon de l'imagination sans objet en vue de développer un art empreint de rationalisme se bornant à " propager " des idées "généreuses", ou un art sollicitant les sentiments et l'imagination à des fins d'endoctrinement (Saint-Simon, 1875). La " puissance des arts », les « armes » du discours sont des plus efficaces. Pour « répandre des idées neuves parmi les hommes, nous les inscrivons sur le marbre ou sur la toile; nous les popularisons par la poésie et le chant ; nous employons tour-à-tour la lyre ou le galoubet, l'ode ou la chanson, l'histoire ou le roman; la scène dramatique nous est ouverte, et c'est là surtout que nous exerçons une influence électrique et victorieuse ", écrivait Saint-Simon.

La poésie de Pierre Lachambeaudie semble avoir réalisé ce programme. Ses Fables (1855) sont précédées d'une préface de Béranger et illustrées. Le poème liminaire dédicacé à Lachambeaudie, de la part d'Antoine-Marc Monnin, fait de l'auteur des fables saint-simoniennes un "apôtre " dévoué à la diffusion de "L'Évangile nouveau »" La fable sert tantôt les idées saint-simoniennes sur le progrès, tantôt la cause de la liberté. Elle véhicule leçons politiques et valeurs fraternelles. Parmi ces thèmes, l'éloge du peuple et la promesse d'un avenir radieux ${ }^{13}$; la loi de la solidarité et de la réciprocité, observée d'après la liaison solidaire de « La vigne et l'ormeau » (Lachambeaudie, 1855 , p. 19) ; l'éloge de la folie en tant que faculté propre aux utopies ${ }^{14}$; ou la fatalité du progrès. « La locomotive et le cheval » conclut ainsi :

La routine au progrès veut disputer l'empire ;

Le progrès toujours marche, et la routine expire. (Lachambeaudie, 1855, p. 13)

D’autres textes de Lachambeaudie, révolutionnaires, insistent sur la liberté (1855, p. 30) plus que sur les principes de cohésion sociale, à la manière des pamphlets qui font alors entendre la voix du pauvre dans les goguettes et caveaux. La veine anarchiste est présente dans la fable "Le gland et les pots ", publiée dans Le banquet des travailleurs socialistes (1849, p. 30). Cette allégorie assimile les chênes maladifs poussant dans un pot aux enfants éduqués selon des contraintes trop strictes. Enfin, "Mes rêves ${ }^{15}$ est la synthèse utopique et le résultat de ce double idéal révolutionnaire et réformiste. Le texte conjugue des images de destruction opérée par le peuple et des visions d'harmonie.

12. « Le poète ». Lachambeaudie (1855), p. 14.

13. Au peuple, Lachambeaudie conseille l'espoir :

Dans les sentiers mauvais tu saigneras encor.

Garde que ton courage aux cailloux ne se brise :

Bientôt tu parviendras à la terre promise

Où doit briller pour tous un nouvel âge d'or. « Le voyageur ", Lachambeaudie (1855), p. 16.

14. "Sur la réalité, malheureux qui s'appuie/ Ah, plutôt embrassons quelque fraîche utopie [...]». « La folle», Lachambeaudie (1855), p. 14.

15. Le caveau, $1^{\text {er }}$ janvier 1842, p. 279-280. [recueil collectif mensuel publié par la société de chansonniers dite « Le caveau»]. 
Dans ce corpus essentiellement engagé et didactique, le peuple s'empare de sa propre cause. Les formes populaires de la fable et de la chanson sont intégrées aux pratiques sociales de la génération 1830, qui suit les traces de chansonniers progressistes, tel Béranger, mais évolue vers l'extrême gauche pendant la décennie 1840. L'entreprise est en elle-même le témoignage d'un progrès, d'une conquête de la parole au cours de l'apprentissage de la démocratie, l'une des gageures du XIX ${ }^{e}$ siècle. Sur le plan de l'art, le renouveau vient essentiellement ici de la thématique sociale, qui, dans la fable, se substitue aux thèmes des moralistes attachés à la compréhension du cœur. Il n'appartient pas à la critique universitaire de juger ces textes mais force est de constater la fantaisie et le piquant de la verve populaire, tandis que l'art social de Lachambeaudie se recommande par les élans d'un cœur sensible et empreint de compassion. On note la variété de ses procédés d'écriture - symbole, parabole, allégorie - relevant du style symbolique.

\section{Le poème de l'utopie saint-simonienne}

Les réformateurs sociaux du XIX ${ }^{\mathrm{e}}$ siècle s'accordent à faire de la représentation utopique elle-même un poème architectural, une œuvre urbaine aux formes parfaites, qui prend sens au cœur du vaste système sémiologique de la création, dans la continuité des méditations de Bernardin de Saint-Pierre sur la nature et la société (Études de la nature, 1784). "L'architecture est pensée durant la première moitié du XIX ${ }^{e}$ siècle comme apte à changer les rapports sociaux. Tout invite donc à un parallèle entre les écritures utopiques, celles de la pierre et celles du papier » (Sylvos, 2007). Calculs de l'attraction passionnée et méditations sur les formes urbaines s'allient avec des songes et visions inspirés à Charles Fourier et à ses disciples, en quête des secrets mystiques de l'univers sur lesquels la société humaine devrait se modeler. Ainsi, le côté maléfique de la nature traduit pour Fourier l'état d'imperfection dans lequel stagne encore la « civilisation ». Les « horreurs de la création » révèlent le dérèglement des passions dans la période civilisée ; les serpents sont le hiéroglyphe de la calomnie et la maladie de la pierre est éloquente au sujet du matérialisme du XIX ${ }^{e}$ siècle. À l'inverse, les créatures du futur - anti-lions et anti-requins - seront à l'image de l'achèvement harmonieux de l'évolution historique, sociale et naturelle (Vergez, 2007). Charles Fourier élabore donc avant la lettre une théorie des correspondances appliquée aux rapports entre la nature et la société, entre le microcosme humain et l'univers.

Dans le fouriérisme comme dans le saint-simonisme, le secret de l'harmonie tient à des « rapports", à des proportions. Les philosophies sociales se veulent des sciences qui superposent les conceptions musicales et littéraires avec des équations ou avec la combinatoire sociale idéale. Les saint-simoniens recherchent des passerelles entre les mathématiques, la langue et la musique : 
Plus tard, en examinant leurs combinaisons, et les diverses formules autour desquelles se groupent, par catégories distinctes, les tournures que revêt par la parole, la pensée humaine, nous rechercherons les analogies qui existent entre les FORMULES ALGÉBRIQUES, c'est à dire la langue TRANSCENDANTALE, ET les TROPES ou FORMULES VERBALES, et aussi les ressemblances de ces dernières avec les FORMES géométriques; ces recherches sont d'un haut intérêt, parce qu'elles supposent ou confirment le lien qui existe entre les trois aspects du langage : le langage INDÉFINI et le langage DÉFINI, par formule ou par forme, selon l'esprit ou selon les sens ; ou en d'autres termes entre la NOTATION MUSICALE, le chiffre, et le signe alphabétique ou hiéroglyphique : lien senti de tous temps, dont le chiffre romain, par sa double signification, littérale et numérique, est un indice, et dont la tentative de Rousseau pour la NOTATION numérique de la MUSIQUE est un autre signe. (Enfantin, 1832, p. 77-78)

Le nombre d'or de la cité parfaite ou l'équation du bonheur est le fondement abstrait des réalisations utopiques selon Enfantin. Ce dernier décèle une correspondance parfaite entre la formule et la forme, l'algèbre et la géométrie et utilise des équations mathématiques en tant que symboles :

Dans ces figures [les courbes élémentaires, asymptote et hyperbole, soumises à deux impulsions différentes, dans la direction de ses axes], la tendance progressive à l'UNION, à l'amoureuse osculation, dans les surfaces et dans les lignes correspondantes, est une IMAGE assez vivante de l'HARMONIE PROGRESSIVE de l'humanité et du monde, soit que l'on prête, dans l'un ou l'autre système, à l'humanité les propriétés coniques ou hyperboliques, soit qu'on les affecte réciproquement au monde. Ce style FIGURÉ fera sans doute sourire et ceux qui par ignorance ne sauraient en avoir l'intelligence, et ceux qui, possesseurs des matériaux de la science, ne concevront pas toutefois que l'on puisse en faire un usage auquel ils n'ont pas songé, usage dont ils ne comprendront ni la valeur encyclopédique, ni la puissance pratique, ni l'inspiration POÉTIQUE ou RELIGIEUSE. (Enfantin, 1832, p. 18)

Loin de pouvoir être envisagées indépendamment, les disciplines ont entre elles, selon les saint-simoniens, de profondes analogies, si bien que la réforme sociale est à la fois science, poétique et religion. À cette série de disciplines, on peut ajouter la musique et l'architecture, qui sont les pierres de touche de l'harmonie sociale. Enfantin marche sur les traces de Pythagore (1832, p. 18), qui représente cette tendance à considérer les formes harmonieuses comme la traduction de formules secrètes.

Quelques poètes exceptionnels ont su concilier le lyrisme et la philosophie grâce à une pensée qui leur est personnelle, mais aussi grâce au pouvoir de transmutation de l'imagination et à une intensité d'émotion qui transcende le didactisme ${ }^{16}$.

16. Charles Baudelaire mentionne la "pente de la rêverie » dans les Feuilles d’automne et La légende des siècles. 
L'exaltation lyrique vient parfois animer le discours humanitaire. "La poésie utopique associe un genre traditionnellement narratif et descriptif à l'harmonie musicale de la prose lyrique. Le recours au lyrisme n'a rien de surprenant tant le saint-simonisme se considère comme une religion. Le nouveau christianisme saint-simonien développe un véritable culte de l'humanité et du progrès, qui, dans La ville nouvelle de Charles Duveyrier, se cristallise autour d'un éloge de la ville anthropomorphe " (Sylvos, 2021, p. 211).

La ville nouvelle ou le Paris des saint-simoniens, de Charles Duveyrier ${ }^{17}$, est l'un des premiers poèmes en prose urbains, rédigé plus de trente ans avant Le spleen de Paris. Contrairement à la poésie versifiée de type didactique, il dénote une volonté d'originalité formelle qui est à la mesure du futurisme novateur de l'anticipation. Surnommé «Le poète de Dieu », Charles Duveyrier, qui était aussi librettiste, se charge de développer, présenté comme suit, le projet du saint-simonien Prosper Enfantin, son maitre :

En vous parlant des travaux industriels, j'ai donc plutôt en vue ceux qui ont pour but direct les jouissances de l'homme, que ceux qui tendent directement à rendre le monde plus productif, je parlerai plutôt de la construction des VILLES et des communications des villes entre elles, que de la culture du SOL. Et voilà pourquoi j'ai choisi l'ARCHITECTURE [...]. (Enfantin, 1832, p. 20)

La foi saint-simonienne se présente, dans Le livre nouveau (1832) comme une religion révélée dont le « Père » Enfantin serait le prophète :

Pour que ces PROJETS de TRAVAUX fussent susceptibles de réalisation, il faudrait en effet que la CONCEPTION fût de nature à se TRADUIRE en un MODĖLE CAPITAL, servant d'exemple et fournissant une INSPIRATION continuelle par le CULTE dont il serait environné, pour tous les TRAVAUX dont ce modèle serait le SYMBOLE. Or la CAPITALE du MONDE nouveau, du royaume humanitaire, la MÉTROPOLE de la FOI UNIVERSELLE, est ce modèle, car c'est le point d'où part toute DIRECTION de grands TRAVAUX sur le GLOBE entier. Voici la CITÉ de DIEU, la JÉRUSALEM nouvelle. ARCHITECTES ! quelle doit être sa forme ? La plus VIVANTE, la plus propre à INSPIRER à l'homme, l'AMOUR, la CONNAISSANCE et la PRATIQUE de ce que l'HOMME doit faire dans le MONDE ; La FORME HUMAINE VITRUVE l'avait RÊVÉE. Et maintenant, ENFANTS, que MICHEL dessine ma PAROLE, et que CHARLES la colore [...]. (Enfantin, 1832, p. 21)

Barthélémy Prosper Enfantin trace le plan d'une entreprise collective dont ses disciples, Michel Chevalier et Charles Duveyrier, seront, chacun pour leur partie,

17. Penseur, écrivain saint-simonien, dramaturge et librettiste surnommé "le poète de Dieu » (Duveyrier, 1832, p. 325). 
les réalisateurs. Michel Chevalier est l'auteur du Système de La Méditerranée tandis que Charles Duveyrier publie la même année, en 1832, La ville nouvelle.

Dans les prolégomènes théoriques mis en place par Le livre nouveau, le symbole ou hiéroglyphe - assure l'articulation entre le poème scriptural et la réalisation urbaine. En effet, si la poésie dessine, colore et célèbre la ville, la forme de la ville anthropomorphe parle de la religion saint-simonienne, de la philosophie humanitaire. Le texte, qui figure, et l'architecture, parlante, échangent leurs caractéristiques. À cette nouvelle religion, humanitaire et panthéiste, prend part la sacralisation du féminin ${ }^{18}$, concrétisée par le temple-femme qui se trouve au centre de la capitale du nouveau monde industriel. L'éloge de la ville passe par la référence au Cantique des cantiques et par l'imitation du style biblique que caractérise la polysyndète (Sylvos, 2021, p. 210).

Mais ce texte tisse aussi, à sa manière, des correspondances entre la terre et le ciel et célèbre leurs harmonies. À la différence du fouriérisme, le saint-simonisme intègre la modernité urbaine et l'industrie au grand Tout. Dans cette vision de La ville nouvelle, l'homme couché, qui donne sa forme à la ville, contient à lui seul l'intégralité du cosmos :

Sa chevelure et sa barbe sont éclairées par un météore de lueurs pâles, qui se jouent dans les massifs, comme l'air et la lumière se jouent dans les cheveux. Ses yeux sont deux soleils tournoyants, éblouissants comme serait mon soleil si je gardais en lui les rayons qu'il disperse dans l'espace, et que je le voulusse montrer seul, quand il fait nuit. De sa bouche séchappe un jet de flammes et d'étincelles qui montent à travers les airs, comme une création d'un monde d'étoiles que ma terre envoie dans mon ciel. (Duveyrier, 1832, p. 343)

La «bouche » de cette ville anthropomorphe est à interpréter au sens propre et au sens figuré. C'est à la fois la bouche de l'homme couché et la bouche d'incendie, et peut-être même le "gueulard», partie supérieure du haut fourneau où sont chargés les minerais et d'où s'échappe le gaz des hauts fourneaux. Si la prose de Duveyrier perd en précision didactique, relativement aux textes ouvriers cités plus haut, elle gagne en puissance d'évocation, en grâce et en poésie, grâce à un style épuré de tout jargon spécialisé. Cet «échantillon » du poème révèle une écriture riche et soignée, qui ouvre la voie au symbolisme poétique. Disqualifié par sa dimension didactique, incompatible avec la poésie selon les critères baudelairiens, Charles Duveyrier réalise toutefois, avant la lettre, l'exigence du grand poète de la modernité, qui rêvait, à travers la métaphore de la "sorcellerie évocatoire " (Baudelaire, 1920, p. 22), une poésie encline à suggérer, plus qu’à dire explicitement. Et ce que retrace cette vision, c'est l'existence d'un échange de type circulaire entre la nature qui fournit

18. Charles Duveyrier voit dans la femme-messie, qui saura trouver des alternatives à la violence martiale de l'homme, l'espoir de l'humanité (Duveyrier, 1832, p. 319). 
l'énergie, le cosmos, d'une part, et la re-création urbaine et industrielle de la modernité. L’échange se traduit syntaxiquement par le passage de la voie passive (" sont éclairées ») à la voix active (« les rayons qu'il disperse dans l’espace») pour désigner le rapport entre l'univers et la ville.

Inventeur de nouvelles harmonies libérées du carcan du vers, Charles Duveyrier anticipe sur le rêve baudelairien d'une "prose poétique, musicale sans rime et sans rythme ", idéal fondé sur " la fréquentation des villes énormes » (Baudelaire, 1917, p. V) :

Paris/, ville qui bout tumultueusement/ ainsi qu'une chaudière de cendres,/

2/ $8 / 8$

ville semblable à ton peuple/, comme lui pâle et défigurée !/

$7 /$

8

Tu gis sur les bords de ton fleuve,/

$8 /$

avec tes noirs monuments/ et tes milliers de maisons ternes/,

$7 /$

$8 /$

comme un amas de roches et de pierres/ que le temps rassemble au bassin des vallées, 9/ $11 /$

et il en sort comme un grondement monotone/ d'une eau comprimée sous ces pierres, $12 /$ $8 /$

ou d'un feu caché/ qui va les crever. (Duveyrier, 1832 p. 324)

$5 /$

$5 /$

Dès la première proposition (« Paris, ville qui bout tumultueusement [...]»), le poète en prose assouplit la syntaxe et se fait le pionnier de cette poésie moderne qui se caractérise par la prédominance du mot (Barthes, 2006, p. 200). «Bloc» ou «pilier » de la strophe, le mot " Paris » est bien libéré ici des « rapports » dans lesquels la poésie classique enchâssait le mot. Il est l'origine, ce qui « nourrit et comble comme le dévoilement d'une vérité » (Barthes, 2006, p. 200). Le poète en prose assouplit aussi le vers et la rime, sans se départir pour autant du sens des harmonies rythmiques et sonores. On retrouve dans cette strophe des bribes d'octosyllables, de décasyllabes ou d'hémistiches parfaits ou approximatifs, à plus ou moins une syllabe près. Le poète en prose assouplit la règle de prononciation du - e. Le - e peut se prononcer selon la diction poétique, ou s'amuïr selon la diction prosaïque. Le lecteur peut donc interpréter à loisir ce texte de nature ambiguë et hybride, selon qu'il apprécie la liberté de la prose ou la régularité des pré-rythmes poétiques (mètres) qui lui sont familiers et qu'il peut retrouver dans les strophes, mais qui prennent vie et sens dans une rythmique originale. Car c'est aussi au mouvement général de la strophe qu'il faut s'intéresser, tant on constate une dilatation, une amplification du rythme qui, depuis des séquences initiales de huit syllabes, tend en fin de strophe vers l'alexandrin et le décasyllabe $(2 \times 5)$. Le rythme se prête à l'évocation d'une énergie archaïque, 
souterraine, source prête à jaillir, volcan révolutionnaire - du peuple ? - ou matière première énergétique de la révolution industrielle, qui déborde la morne apparence de la capitale et sa temporalité actuelles.

L'ambition sacerdotale de Charles Duveyrier, qui retrace une nouvelle Genèse plaçant l'homme et ses œuvres au centre du monde et de la cité modernes, se traduit par un souffle épique et une ligne continue, qui reste étrangère à la poétique baudelairienne du fragment, du " petit » poème, du " soubresaut » et du " cri », même si la strophe décompose cette coulée prophétique en blocs ayant leur unité, leur métrique, leur fonction et leur coloration propres. Ici, la matière sonore de la strophe est caractérisée par deux dominantes en finale, celle du [ER] (" chaudière ", " ternes », " pierres », " pierres ») et celle du [e] (" défigurée », " milliers ", " vallées », " comprimée », " caché », " crever »), après un début qui harmonise «tumultueusement », « cendres » et « monuments », puis « peuple » et « fleuve ».

Le poème de Duveyrier s'inscrit dans la ligne tracée par Saint-Simon, persuadé de sa capacité à fonder un " nouveau christianisme " ${ }^{19}$, qui serait à l'aune de la "nouvelle Genèse » d'un globe redevenu paradis ${ }^{20}$. Alors que d'antiques cosmogonies poétiques, tel l'épicurisme d'un Lucrèce, faisaient advenir d'en haut tout ce qui se passait sur la terre en vertu de la théorie des atomes, la terre et les hommes acquièrent la capacité d'engendrer un "nouveau monde ${ }^{21}$. Elle procure un nouveau souffle à la création, un souffle venu des entrailles de la terre, en écho à l'imaginaire des profondeurs qui caractérise la psyché romantique (Richard, 1955). On reconnaît bien là le caractère démiurgique des religions modernes, nourries par un imaginaire chthonien et technocratique. Il entre évidemment en résonance avec la réalité des complexes industriels, de la sidérurgie et de la métallurgie qui transforment la matière grâce à de nouveaux procédés mis au point en Angleterre dès le XVIII siècle $^{22}$.

Le deuxième volet de cette étude s'est attaché à différencier la prose lyrique saint-simonienne de la poésie sociale en vers marquée par le didactisme, comme l'avait montré la première partie de cette contribution, qui toutefois souligne les particularités attachantes, la fantaisie et la vigueur de la poésie populaire. La ville nouvelle porte

19. Ouvrage de Saint-Simon publié en 1825.

20. «L'âge d'or qu'une aveugle tradition a placé jusqu'ici dans le passé, est devant nous », tel est l'épigraphe aux Opinions littéraires, philosophiques et industrielles, projet d'ouvrage de 1825 dont Le Nouveau Christianisme n'est qu'un extrait. Dès les Lettres de 1803, Saint-Simon n'avait-il pas « rêvé » à ces paroles de Dieu lui déclarant qu' « un jour viendra que je ferai de la terre un paradis » $(1803, \mathrm{I}, 48)$; « autrement dit que le paradis serait terrestre et dans le futur, et non plus céleste et au-delà ?» (Musso, 1999, p. 95).

21. C'est aussi le titre de la somme de Fourier intitulée Le nouveau monde industriel et sociétaire (1829).

22. Sur trois générations, la famille de quakers britanniques Darby révolutionne la sidérurgie et la métallurgie. Ce sont la fabrication de la fonte à partir du coke, moins coûteux que le charbon de bois, le pudlage, qui permet d'obtenir du fer à partir de la fonte et, enfin la fabrication du premier pont métallique, Iron Bridge (1779; cf. aussi le pont d’Austerlitz édifié en 1807). 
bien un enseignement, comme le suggère le recours aux symboles ${ }^{23}$. Enseigner, c'est insignare, faire connaitre par un signe et c'est bien ce que font les saint-simoniens, dévoilant la religion de l'humanité à travers l'emblème urbain de l'homme couché. Malgré cette donnée qui, aux yeux de Baudelaire, parait condamner La ville nouvelle au prosaïsme, cet enseignement est sublimé par le souffle épique et lyrique de la prophétie, par une intention novatrice qui s'attache au progrès social comme à la forme de la ville et à celle de son poème. Les micro-lectures de La ville nouvelle proposées dans la deuxième partie de cet article tendent à définir l'apport de la poétique saint-simonienne au processus évolutif qui, au cours du XIX ${ }^{\mathrm{e}}$ siècle, achève d'émanciper la poésie du vers lors de la lente émergence du poème en prose, depuis Les chansons madécasses de Parny (1787) jusqu'aux petits poèmes en prose de Baudelaire (1869), en passant par Gaspard de la nuit (1842). Dans ce poème, le désir de faire signe ne semble pas incompatible avec le sens du rythme (Meschonnic, 1999). L'art social crée une ville à l'image du culte humanitaire. L'apologie du peuple se met en place. La capitale de la France, recréée par et pour le peuple, se dit dans une prose assouplie, dans une prosodie originale. La différence entre poètes didactiques et poètes visionnaires tient à l'écart entre discours rationnel et prose lyrique. Avec Duveyrier, la description et le lexique spécialisé de l'industrie, présents dans la poésie ouvrière et sociale en vers, qui prolonge la tradition didactique et philosophique classique pratiquée depuis Empédocle jusqu'à l'abbé Delille, laisse place à un art suggestif qui, pour réhabiliter la matière, s'appuie sur les effets sonores, rythmiques et visuels originaux du poème en prose.

Cette réflexion tend à mettre en relief la fécondité de la Révolution industrielle qui renouvelle l'imaginaire et appelle de nouvelles formes, à l'instar de la Révolution politique de 1789 que l'École d'Iéna (1799) considérait comme une fracture définitive dans l'histoire de la poétique (Labarthe, 1978, p. 310). Un degré supplémentaire dans la sublimation du poème industriel est franchi avec la prose poétique et supernaturaliste de Nerval, qui, dans le Voyage en Orient, évoque en un curieux télescopage temporel du retour aux origines de la Genèse et de la métallurgie, la flore métallique du « monde souterrain ", les forges intérieures de la terre creuse, mundus inversus où règnent les ouvriers, dirigés et ralliés par la lignée des fils de Caïn (Sylvos, 1997, p. 134-139). C'est ensuite, dans Aurélia, un étrange lama de métal et de feu, un atelier où l'on recrée une pseudo-humanité venue d'en bas et non plus d'en haut, comme est supposée lêtre la créature engendrée par Dieu. Mais un dialogue y conclut aux limites de ces chimères modernes qui ne sont que la recréation industrielle et artificielle de la vie :

Ces fleurs qui vous paraissent naturelles, cet animal qui semblera vivre, ne seront que des produits de l'art élevé au plus haut point de nos connaissances, et chacun les jugera ainsi. (Nerval, 1985, p. 65-66)

23. «Osez donc être les précepteurs de l'humanité, dirons-nous aux artistes, et apprenez de SaintSimon ce qu'il faut aujourd'hui lui enseigner » (Barrault, 1830, p. 76). 
Ici, l'imaginaire social et industriel est totalement découplé du didactisme, sans pour autant sombrer dans un esthétisme gratuit. Le pacte implicite repose sur une confiance absolue dans le pouvoir des associations poétiques et de la sagacité du lecteur à faire entrer le texte en résonance avec son actualité.

Il peut paraittre aberrant aujourd'hui d'envisager la naissance de l'industrialisme et la modernité urbaine sous l'angle de la fertilité et de la naissance, alors que la société actuelle subit les conséquences du potentiel destructeur de la surnature technologique sur la nature elle-même, depuis le passage à l'anthropocène amorcé par la Révolution industrielle. Cette période d'enthousiasme pro-industriel durera peu, optimisme représentatif du printemps du romantisme, qui s'actualise dans d'autres textes tel Le roman de lavenir de Félix Bodin (1834) ou Paris révolutionné de Louis Desnoyers (1834), à un moment où l'on découvre les charmes de l'anticipation, où le techno-imaginaire semble riche de promesses créatives ouvrant de " nouvelles routes pour la littérature, de nouveaux champs pour l'imagination » (Bodin, 1834, p. 29) sans que l'industrie et la banque aient encore dévoilé leurs travers. Cette période se caractérise par l’émergence d'un « techno-messianisme », déjà miné par le « techno-catastrophisme » (Musso, 2019-2020, p. 117) dont la naissance prend place quelque part entre Le dernier homme de Cousin de Grainville (1805), le Frankenstein de Mary Shelley (1818) et Le monde tel qu'il sera d'Émile Souvestre (1846).

\section{RÉFÉRENCES}

Barrault, É. (1830). Aux artistes. Du passé et de l'avenir des Beaux-Arts. Doctrine de SaintSimon. Paris : Alexandre Mesnier.

Barthes, R. (2006). Y a-t-il une écriture poétique ? Le Degré zéro de l'écriture, dans Euvres complètes. Éric Marty (éd.). Tome I (1942-1961). Paris : Seuil.

Baudelaire, Ch. (1868). Salon de 1859. Dans Curiosités esthétiques. Paris : Michel Lévy.

Baudelaire, Ch. (1917). Lettre à Arsène Houssaye. Le spleen de Paris. Paris : Émile Paul.

Baudelaire, Ch. (1920). Journaux intimes, Fusées, mon cœur mis à nu. Paris : Adolphe Van Bever.

Breton, A. (1999). Ode à Charles Fourier. Signe ascendant et autres textes. Paris : Gallimard.

Bodin, F. (1834). Le roman de l'avenir. Paris : Lecointe et Pougin.

Caraion, M. (2016). Ironie et grandiloquence du progrès. Dans C. Barel-Moisan, A. Déruelle et J.-L. Diaz (éd.). Actes du VIIe Congrès de la SERD. Le XIX ${ }^{e}$ siècle face au futur. Penser, représenter, rêver l'avenir au XIX ${ }^{e}$ siècle. https://serd.hypotheses.org/1879

Combe, D. (2005). Le poème philosophique ou «l'hérésie de l’enseignement ». Études françaises, 41(3), 63-79.

De Liefde, C. L. (1927). Le Saint-simonisme dans la poésie française entre 1825 et 1865. Haarlem : Drukkerij « Amicitia ».

Du Camp, M. (1860). Les Chants modernes. Paris : Librairie nouvelle.

Duveyrier, Ch. (1832). La ville nouvelle ou le Paris des saint-simoniens dans Le Livre des centet-un. Paris : Ladvocat. 
Enfantin, P. B. (1832). Le Livre nouveau, mis en ligne par l'École alsacienne, d'après Gallica. https://archives.ecole-alsacienne.org/CDI/pdf/1301/130136_SIM.pdf

Gille, Ch., Letac, A. et Berthier, E. (dir.). (1846). Les cent et une petites misères, rédigée par les meilleurs chansonniers de l'époque. Paris : Letac.

Lachambeaudie, P. (1855). Fables. Paris : Jean Bry Libraire-Éditeur.

Lacoue-Labarthe, P. et Nancy J.-L. (1978). L'absolu littéraire. Paris : Seuil.

Le caveau ( $1^{\mathrm{er}}$ janvier 1842). Recueil collectif mensuel publié par la société de chansonniers dite «Le caveau », 279-280.

Meschonnic, H. (1999). Manifeste pour un parti du rythme. En ligne. http://www.berlol.net/ mescho2.htm

Musso, P. (1999). Saint-Simon et le saint-simonisme. Paris : Presses universitaires de France.

Musso, P. (2019-2020). Le désir technologique de Dieu. Quaderni, 99-100, 113-124.

https://doi.org/10.4000/quaderni.1567

Nathan, M. (1981). Le Ciel des fouriéristes. Habitants des étoiles et réincarnations de lâme. Lyon : Presses universitaires de Lyon.

Nerval, G. de. (1985). Aurélia. Paris : Lachenal et Ritter.

Poe, E. (1887). Principes de la poésie (F. Rabbe, trad.). Paris : Albert Savine.

Racault, J.-M. (1991). L'Utopie narrative en France et en Angleterre 1675-1751. Oxford : The Voltaire Foundation.

Richard, J.-P. (1955). Poésie et profondeur. Paris : Seuil.

Saint-Simon, H. de. (1803). Les Lettres d'un habitant de Genève à ses contemporains. s.l. : s.n.

Saint-Simon, H. de. (1875). L'artiste, le savant et l'industriel. Euvres complètes de Saint-Simon et d'Enfantin, vol. 10. p. 201-258. Extrait p. 209-213. https://books.openedition.org/inha/5083?lang=fr

Sylvos, F. (1998). Nerval ou l'antimonde. Discours et figures de l'utopie, 1826-1855. Paris : L'Harmattan.

Sylvos, F. (2006). Le paysage urbain dans deux utopies sidérales du XIX ${ }^{\mathrm{e}}$ siècle. Crée, 1. S. Meitinger et Ch. Chelebourg (dir.). 55-72.

Sylvos, F. (2007). Sémiologie de l'utopie en France (1800-1850). Fabula / Les colloques, Séminaire "Signe, déchiffrement, et interprétation ». http://www.fabula.org/colloques/ document907.php

Sylvos, F. (2008). Lépopée du possible ou l'arc-en-ciel des utopies. Paris : Honoré Champion.

Sylvos, F. (2014). Introduction. TrOPICS, 2. "Théâtre et utopie ». 1-12. https://tropics. univ-reunion.fr/accueil/numero-2/i-theatre-et-utopie/introduction-francoise-sylvos

Sylvos, F. (2015). Rire des Rouges en 1848. Une approche transgénérique et plurimédiatique. Dans C. Dessy et V. Stiénon (dir.), (Bé)vues du futur. Les imaginaires visuels de la dystopie (1840-1940) (p. 93-106). Villeneuve-d'Ascq : Presses universitaires du Septentrion.

Sylvos, F. (2021). Voyage en ballon au-dessus des utopies et anticipations. Poétique et idéologie, du premier au second $\mathrm{XIX}^{\mathrm{e}}$ siècle. Francofonia, 81. L'utopie sociale dans la littérature du XIX ${ }^{e}$ siècle. 207-226. https://www.lilec.it/francofonia/81-2021

Trousson, R. (1998). D’utopies et d'utopistes. Paris : L'Harmattan.

Vergez, A. (2007). Initiation à la lecture de Charles Fourier. Consultable en ligne sur le site Charlesfourier.fr. http://www.charlesfourier.fr/article.php3?id_article=379

Verne, J. (1863). Paris au XX siècle. Paris : Hachette/Le cherche Midi. 
RÉSUMÉ : Cet article oppose les textes de propagande positive et ouvrière en vers français du XIX ${ }^{\mathrm{e}}$ siècle (Du Camp) à la prose lyrique et visionnaire des saint-simoniens (Duveyrier). À partir du jugement de Baudelaire sur l'incompatibilité entre poésie et didactisme, on s'interroge sur le statut et l'artialité de la poésie sociale. $\mathrm{Du}$ côté de la poésie ouvrière (Cent et une petites misères, Euvre sociale), on découvre la verdeur de la langue populaire, la vis comica et la fantaisie tandis que la fable socialiste (Lachambeaudie) est remarquable par la compassion. Sous la plume des prophètes du progrès (Enfantin), les disciplines - religion, architecture, poésie, mathématiques - loin d’être cloisonnées, sont objets analogues et langages convertibles. Le poème, l'image et la cité idéale elle-même changent leurs caractéristiques et se correspondent. L'innovation sociale ne peut se dire qu'à travers la forme novatrice du poème en prose urbain et la Révolution industrielle appelle une révolution des formes poétiques. La poéticité de ces textes, inversement proportionnelle au réalisme et à la spécialisation du lexique employé, tient à un art de la suggestion et à l'essor d'un imaginaire renouvelé par la modernité technique, citadine et scientifique.

Mots-clés : XIX ${ }^{e}$ siècle, littérature française, utopie, poésie sociale, poème en prose, didactisme, positivisme

\section{Poetry and utopia in France during the 19th century}

ABSTRACT: This paper confronts versified positivist propaganda (Du Camp) with lyrical and visionary prose of Saint-Simon's followers (Duveyrier). Taking as the point of departure Baudelaire's judgment about the incompatibility between poetry and didacticism, this paper queries the status and aesthetic value of social poetry. The working class poetry (One hundred small miseries, Social work) is full of vitality of the popular style, replete with humorous energy and fantasy, whereas the socialist fable (Lachambeaudie) expresses a lot of empathy. Under the positivist prophets' pen, the disciplines - religion, architecture, poetry, mathematics - are not separated but analogous and convertible. According to these thinkers, there is no difference between a poem, picture of the ideal city and utopia itself. Social innovation can only be told by the innovative form of the urban prose poem, and the Industrial Revolution calls for a revolution of poetic forms. The poeticity of these texts, inversely proportional to the realism and to the specialization of the lexicon used, stems from the art of suggestion and the rise of imagination renewed by technical, urban and scientific modernity.

Keywords: 19 th century, French literature, utopia, social poetry, prose poem, didacticism, positivism 